\title{
THE USE AND MISUSE OF CONFIDENTIAL INFORMATION
}

\author{
By Dr ZAIDa M. Hall \\ Consultant Psychiatrist, Knowle Hospital, Fareham
}

The dilemma

There has been increasing concern about Governmental collection of information for statistical analysis (World Medicine, 13 June 1973; British Medicine, 7 April 1978; the Sunday Times, 2 July 1978). Recently the Central Ethical Committee of the BMA refused to agree that General Practitioners should record information about pre-school children containing details about the mother's obstetric history. Again, the Regional Community Physicians refused to allow Police access to hospital diagnostic information about patients who had been involved in road traffic accidents. Yet, since 1964, psychiatrists have apparently been happy to send to the DHSS on HMR 1(Psych) forms identifiable information containing such highly-sensitive details as whether an inpatient is manic-depressive, homosexual, epileptic, has used alcohol or other drugs, has venereal disease, is living with someone other than his wife, or has been in prison.

There are clear advantages in providing such details for the purposes of resource allocation, forward planning and research, all of which indirectly benefit the patient; but if the details are readily identifiable with a particular patient, there are also disadvantages. Firstly, the confidentiality between patient and doctor has been breached. A patient gives tacit agreement that a doctor should share information with other professionals who take part in his investigation or clinical care, but not that information should be sent to a central Government computer bank for coding. Indeed, it is arguable whether ethically or morally we are justified in so doing. Secondly, although in the past fourteen years of the Mental Health Enquiry there have apparently been no breaches of confidentiality, it is not improbable that, with increasing Government control, information may be transferred from one Government Department to another and consequently be open to misuse. (A year after the last Census, in spite of Government assurances that the information was confidential, ex-nurses found themselves being questioned on why they had left the profession). The attitude of the Government could subtly alter over the years until it assumed the right to use all data as it considered necessary. This could open

*As Chairman of the Knowle Hospital Records and Confidentiality Sub-Committee I have been increasingly involved with matters of confidentiality but I write about the following issues in a personal capacity. up possibilities for misuse without any particular official being consciously corrupt. It might also be possible for a commercial organization to obtain access for corrupt purposes.

Dr Baldwin, Dr Leff and Professor Wing have reported the views of the Royal College of Psychiatrists' Social and Community Psychiatry Group on these matters (British Journal of Psychiatry, (1976), 128, 417-427). They confirm that the names of patients are not needed for resource allocation and planning, but state that they are needed for epidemiological research requiring record linkage. They feel that the highest risks of a breach of confidentiality arise from possible lapses of security locally, and do not think that the storage of identifiable information with the DHSS computer bank is 'likely to be abused'. They agree, however, that psychiatrists should make their own personal decisions whether or not to fill in identifiable data on the forms. Nevertheless, despite these assurances, the doubt remains whether psychiatrists are safeguarding their patients' privacy adequately.

\section{Possible solutions}

About 5\% of psychiatrists have satisfied their consciences in this matter by not filling in the HMR I forms or the name and house or flat number. Others leave the names in but falsify the diagnosis (MIND survey reported in the Sunday Times, 21 August 1977). The Data Protection Committee has done valuable work in formulating procedures to ensure that no improper use will be made of the data stored. Yet, however properly the information is used, the likelihood is that at any rate some patients from both psychiatric and general hospitals would not want the information to leave the hospital and would refuse to allow it if specifically asked for their consent.

In Knowle Hospital the consultants, with one exception, have taken the decision to withhold all identifiable information on HMRI forms. We consider the following information to be identifiable:

- name, address (other than district), date of birth (other than year only) and NHS number;

- names addresses and telephone numbers of relatives.

We therefore omit these details on pages 2 and 3 of the copies of these forms which are sent out of the hospital. This leaves only the hospital reference number as a possible source of identification through the Data Custodian. (I should perhaps add that we are also tightening up security within our hospital and are 
looking into aspects of confidentiality between NHS and non-NHS disciplines).

\section{The computer problems}

However, we are told that our action in withholding this identifiable material raises problems for our own Wessex Regional computer and for the DHSS computer, for the following reasons:

(a) It is difficult to match a patient's admission (page 2 of HMR1) with his discharge (page 3 of HMRI) unless his name and exact date of birth are given, for errors can easily be made in recording the hospital number and the date of admission (the only other exact identification used at present for matching). One pair of mismatched admission and discharge sheets can multiply in a geometric progression, making chaos of the whole statistical analysis.

(b) Resource allocation depends on the locality a patient lives in. The boundary between localities may sometimes divide a street so that, in some instances, it is essential to know the number of a house and the street for accurate coding. With imprecise information the administrators cannot tell, for example, if the Knowle catchment area is importing in-patients from outside and therefore may not allocate enough money.

(c) The complete date of birth may be important for epidemiological research.

The technical difficulties could be resolved by altering methods of computer coding. Since the statistical and computer experts are very conscientious in protecting the confidentiality of material once it is in their care, they are naturally reluctant to see the intrusion into privacy caused by identifiable information leaving the hospital as an important enough issue to make them devise ways of overcoming the technical problems or even do without some information that might prove useful for research-particularly when psychiatrists have apparently already sanctioned the computer use of identifiable information. But the technical problems are not insoluble and could be overcome if the will were there. For instance:

(i) Matching could be accomplished by using additional data, e.g. the locality of an address; or by incorporating a special number into each batch of four duplicate HMR 1 forms, as is done in the Oxford Region.

(ii) Location coding could be checked by telephone by a local computer coder for the particular psychiatric hospital. (This is now done for Knowle
Hospital in the Wessex Region where all the HMR1 information is coded locally before the forms are sent to the DHSS computer. Our local coder is anyway often in touch with our Hospital Records Department concerning undecipherable writing and other ambiguities. The whole transaction is oral and nothing identifiable leaves the hospital in writing). This could only be done if every Region coded locally: at present they have their own computers, but not the staff to undertake full local coding.

Suggested standard procedure May I suggest therefore:

1. That all Regions should code psychiatric forms locally at their Regional Computer Centre, as we do, to ensure uniformity of methods and standards.

This would enable Regional Authorities to allocate resources and check on consumption within their Regions themselves. Because of the ease of telephone communication to exclude ambiguities, the accuracy of the information coded (always the earliest source of error) would almost certainly be improved.

2. That the DHSS should accept local computer analyses. It would then be easy for local doctors (who have been given the confidential information by the patient in the first place) to monitor the precautions taken to ensure confidentiality in a way which would not be possible at a national centre.

3. Most importantly, that all psychiatric hospitals should withhold identifiable material on the Mental Illness Enquiry or HMRI forms.

This paper is a plea to psychiatrists as a whole to reconsider the implications of allowing confidential information about their patients to leave psychiatric hospitals. In the course of time, out-patients as well as in-patients will probably be included in computer data, so that, unless we re-think the whole procedure, as much as one tenth of our population could have very sensitive data about their personal lives recorded in a central data bank without their specific consent. This unsatisfactory situation cannot be rectified by the work of the Data Protection Committee; the right principle must be to prevent sensitive data from reaching computer banks unnecessarily, rather than to devise elaborate procedures for ensuring its confidentiality after it has done so. 